This is an author produced version of a paper published in American Journal of Physiology- Hearth and Circulatory Physiology. This paper has been peerreviewed but does not include the final publisher proof-corrections or journal pagination.

Citation for the published paper:

Rosengren, Bert-Inge and Rippe, Anna and Rippe, Catarina and Venturoli, Daniele and Sward, Karl and Rippe, Bengt.

"Transvascular protein transport in mice lacking endothelial caveolae" American Journal of Physiology- Hearth and Circulatory Physiology, 2006, Issue: Dec 24.

http://dx.doi.org/10.1152/ajpheart.01364.2005

Access to the published version may require journal subscription.

Published with permission from: American Physiological Society 


\section{Transvascular protein transport in mice lacking endothelial}

\section{caveolae}

Bert-Inge Rosengren ${ }^{1,2}$, Anna Rippe ${ }^{1}$, Catarina Rippe ${ }^{1}$, Daniele Venturoli ${ }^{1}$, Karl Swärd $^{3}$, and Bengt Rippe ${ }^{1}$

${ }^{1}$ Department of Nephrology, Lund University, Sweden

${ }^{2}$ Department of Biomedicine, Section of Physiology, University of Bergen, Norway

${ }^{3}$ Department of Experimental Medical Sciences, Lund University, Sweden

\section{Correspondence: $\quad$ Bert-Inge Rosengren \\ Department of Nephrology, \\ University Hospital of Lund, \\ S-221 85 LUND}

Sweden

Fax: $+46+46-2114356$

Tel: $+46+46-171247$

E-mail: Bert-Inge.Rosengren@med.lu.se

Running head: Transvascular protein transport independent of caveolae 


\begin{abstract}
Caveolae are $\Omega$-shaped vesicular structures postulated to play a role in transvascular protein transport. Studies on mice lacking endothelial caveolae, caveolin-1 knock out mice (cav-1-KO), indicate increased macromolecular transport rates. This was postulated to be due to the appearance of an alternative pathway. The present study tested if an alternative pathway had appeared in cav-1-KO. Male cav-1-KO ( $\mathrm{n}=12)$ and male control mice $(\mathrm{n}=13)$ were intubated and anesthetized using $2 \%$ isofluran. ${ }^{125} \mathrm{I}$-albumin, ${ }^{131} \mathrm{I}-$ immunoglobulin M (IgM) and polydisperse FITC-Ficoll were administered i.v. During tracer administration, a 90 min peritoneal dialysis dwell was performed. Clearance of tracers to dialysate, and permeability-surface area product (PS) for glucose were assessed. Transvascular protein transport was higher in cav-1-KO compared to control. Albumin clearance from plasma to peritoneum was $0.088 \pm 0.008$ $\mu \mathrm{l} / \mathrm{min}$ in control, and $0.179 \pm 0.012 \mu \mathrm{l} / \mathrm{min}$ in cav-1-KO $(\mathrm{p}=0.001)$. IgM clearance was $0.049 \pm 0.003$ $\mu \mathrm{l} / \mathrm{min}$ and $0.083 \pm 0.010 \mu \mathrm{l} / \mathrm{min}$ in control and cav-1-KO, respectively $(\mathrm{p}=0.016)$. Ficoll clearance was increased in cav-1-KO mice. In conclusion, the lack of caveolae in cav-1-KO resulted in a marked increase in macromolecular transport. A two-pore analysis of the Ficoll clearance data revealed that the higher transport rate in cav-1-KO was not compatible with the appearance of an "alternative" pathway for macromolecular transport. In contrast, the higher transperitoneal protein and Ficoll clearance is consistent with passive porous transport through an unperturbed two-pore system, presumably at an elevated capillary hydraulic pressure. Alternatively, the data may be explained by reductions in the selectivity of the endothelial glycocalyx, leading to an increased capillary hydraulic conductivity and large solute filtration.
\end{abstract}

Key words: permeability; pores; transcytosis; protein; vesicles 


\section{Introduction}

How the transfer of macromolecules occurs across capillary walls has been vividly debated over the past few decades. The two opposing views are: 1) that macromolecules are shuttled in plasmalemmal vesicles (caveolae) back and forth across the capillary endothelium by transcytosis; or 2) that the transfer is completely passive, in the form of convective protein passage across large pores, located either paracellularly or transcellularly, i.e. porous transport. The question is not whether the process of transcytosis exists or not, rather its quantitative importance. Transcytosis is energy-requiring and specific and would logically be reserved for specialized processes, and not for the bulk transport of proteins across the endothelial barrier. Endo- and exocytosis play important roles in the cellular uptake and release of various proteins and polypeptides as well as the insertion and deletion of proteins in the plasma membrane. Michel and Curry (12) and Rippe et al. (27) have written recent reviews on the controversy.

The evidence in favor of endothelial transcytosis is to a large extent based on morphological studies $(15,19,22,37,38)$. There is, however, strong evidence that a vast majority of the apparently "free" plasmalemmal vesicles seen in routine electron microscopic sections are actually part of complex invaginations of the endothelial cell membrane (1), raising doubt about the classic "shuttle" hypothesis (8). Furthermore, since transcytosis must be a temperature dependent transport mechanism, it should be highly sensitive to temperature reductions. However, tissue cooling did not reduce albumin transport more than could be predicted by the temperature induced increases in viscosity following cooling $(24,33,35)$. Moreover, porous transport through water-filled channels is dependent upon hydraulic pressure, whereas transcytosis would not to be primarily coupled to hydrostatic pressure. Since several studies have indicated a pressure-dependence of the transcapillary passage of albumin, and to some extent of lowdensity lipoprotein (LDL), this again supports the contention that macromolecules are transported via porous pathways, and not via vesicles $(24,35,36)$. Other attempts to study the importance of transcytosis in protein trafficking include the use of chemicals that interfere with transcytosis dynamics, such as the transcytosis inhibitors N-ethylmaleimide (NEM) and filipin. It was suggested that NEM markedly inhibits the transvascular transport of small proteins and albumin in cell cultures in vitro and also in in situ 
perfusion experiments $(20-22,37,38)$. These results have, however, been seriously questioned, since we and others have clearly demonstrated that NEM induces toxic endothelial damage in rat lung, aorta, peritoneum and skeletal muscle $(4,13,30,32)$, whilst it was not able to inhibit transcytosis in non-toxic concentrations.

The latest approach in the study of transendothelial protein transport is the use of caveolin-1 knock out (cav-1-KO) mice. Caveolae are 50 to $100 \mathrm{~nm}$ wide $\Omega$-shaped plasma membrane invaginations. Since their first description some 50 years ago (14), they have been ascribed many functions, including signaling, interactions with enzymes, and protein transport via transcytosis. Caveolin is the principal marker for caveolae. The mammalian caveolin gene family codes for caveolin-1, -2 , and -3 . Caveolin-1 and -2 are coexpressed and are frequently found in many cell types including endothelial cells. Caveolin-3 expression, however, is muscle-specific and found in heart, skeletal and smooth muscle. Caveolin-1 deficient mice do not form endothelial caveolae, but the mice are viable, fertile and appear largely normal (6). Schubert and coworkers (39) recently published the first attempt to study transvascular protein transport in cav-1-KO mice and suggested the mice to be hyperpermeable to albumin. The authors speculated that this was due to the de novo appearance of a transport route across the endothelium in the cav-1-KO mice, compensating for the lack of transcytosis.

In the present study we show that, despite the loss of transcytosis, macromolecular transport is higher in cav-1-KO mice, but not due to the presence of an alternative paracellular pathway. We conclude that transvascular transport of macromolecules is compatible with passive filtration across large pores. The higher macromolecular transport rates in cav-1-KO mice is conceivably due to a higher filtration rate of macromolecules through large pores, driven by an elevated capillary hydraulic pressure. The higher capillary pressure may be due to precapillary vasodilatation caused by the increased eNOS activity in the cav-1-KO mice. Alternatively, the data may be explained by a reduced selectivity of the endothelial glycocalyx in cav-1-KO mice, giving rise to an increased capillary hydraulic conductivity and large solute filtration. Such modifications may arise in response to the higher NO levels in cav-1-KO mice (7). Furthermore, we supply the first data on hemodynamic parameters in cav-1-KO mice. 


\section{Materials and Methods}

Experiments were performed on 12 male cav-1-KO mice with a C57BL/6 background (The Jackson Laboratory, Bar Harbor, ME) having an average body weight (BW) of $27.8 \pm 0.6$ g (SEM) and 13 age matched male C57BL/6 mice (Møllegaard, Copenhagen, Denmark) as controls (BW $28.3 \pm 0.6 \mathrm{~g}$ ). The mice had free access to food and water until the day of experiment. The investigation was conducted in conformity with the APS's Guiding Principles in the Care and Use of Animals.

\section{General surgery and anesthesia}

Anesthesia was induced by placing the mice in a small plastic cage containing $4 \%$ isofluran (Isofluran Forene, Abbot Scandinavia AB, Solna, Sweden) in room air using an Univentor 400 Anaesthesia unit (Univentor Ltd., Zejtan, Malta). The mice were then transferred to a heating pad (Temperature Control Unit HB 101/2, Panlab s.I., Barcelona, Spain) where body temperature was kept constant at $37 \pm 1{ }^{\circ} \mathrm{C}$ with a rectal probe, and the fur over the abdomen was closely shaved. While maintaining anesthesia using a small mask covering the nose, a tracheotomy was performed. After intubation, anesthesia was maintained and controlled using 2\% isofluran in room air and a small animal respirator (Mouse ventilator 28025, Ugo Basile, Comerio, Italy). Tidal volume was set at $0.35 \mathrm{ml}$ and a positive end-expiratory pressure of 5-6 mmHg was applied to prevent pulmonary atelectasis. Right jugular vein was cannulated for infusion of saline and for administration of tracers. Left femoral artery was cannulated for blood sampling and for continuous monitoring of blood pressure on a polygraph (Model 7B; Grass Instruments Co., Quincy, MA). A catheter (Neoflon $\varnothing 0.7 \mathrm{~mm}$, Becton Dickinson Infusion Therapy AB, Helsingborg, Sweden) was inserted into the lower right quadrant of the abdominal wall for dialysis fluid infusion and sampling.

\section{Peritoneal dialysis protocol}

In peritoneal dialysis (PD) a hyperosmotic solution is instilled into the peritoneal cavity to remove excess fluid and metabolites from the blood of patients with impaired renal function (28). In this study, PD was performed in control and cav-1-KO mice using $2.5 \mathrm{ml}$ of PD fluid (Gambrosol Trio ${ }^{\circledR}$, Gambro, Lund, Sweden), which essentially is a lactated Ringer solution containing $1.5 \%$ glucose as the osmotic agent (osmolarity $357 \mathrm{mOsm} / \mathrm{l}$ ). The peritoneal volume was chosen by scaling down the corresponding dialysis 
volume from man. However, a negotiation between scaling versus body weight and body surface area was applied to achieve a volume large enough for reliable measurements, without raising the intraperitoneal pressure significantly. The dialysis fluid was warmed to $37^{\circ} \mathrm{C}$ in a heating bath prior to infusion. The mice were allowed to stabilize for at least 20 minutes after surgery. $0.1 \mathrm{MBq}$ RISA $\left({ }^{125}\right.$ I-human serum albumin; Isopharma, Kjeller, Norway) and $0.1 \mathrm{MBq}{ }^{131}$ I labeled immunoglobulin $\mathrm{M}(\operatorname{IgM})$ were given as a bolus dose in the right jugular vein at the onset of the dwell to assess their clearance $(\mathrm{Cl})$ from plasma to dialysate. Blood samples were drawn at $0,5,10,20,40,60$, and 90 min of the dwell $(10 \mu 1$ for analysis of radioactivity and $3 \mu 1$ for blood glucose determination). In addition, $40 \mu 1$ of blood were drawn for measurement of hematocrit at 5, 60, and 90 min of the dwell. Dialysate was sampled at $0,5,10,20,40$, 60 , and $90 \mathrm{~min}$ of the dwell ( $50 \mu 1$ for analysis of radioactivity and $25 \mu 1$ for glucose measurements). After completion of the dwell period (90 $\mathrm{min}$ ), the peritoneal cavity was opened and the dialysis fluid was totally recovered using a syringe and pre-weighed gauze tissues. Dialysate volume, as well as the albumin clearance from peritoneum to plasma (as a marker of direct lymphatic absorption), were assessed from separate experiments using RISA as a volume marker ( 5 controls and 4 cav-1-KO mice) (40). The average dialysate volume vs. time curve and the albumin clearance from peritoneum to plasma, for respective strain, was then used for clearance calculations and for calculation of permeability-surface area product (PS).

\section{Tracer labeling and characterization}

Ficoll was labeled with fluorescein isothiocyanate (FITC) (Sigma, St. Louis, MO). 0.1g of Ficoll was dissolved in $1 \mathrm{ml}$ dimethyl sulfoxide (DMSO, Sigma). $2 \mathrm{mg}$ sodium bicarbonate and $10 \mathrm{mg}$ of FITC were added and the solution was heated for 15 minutes at $100^{\circ} \mathrm{C} .20 \mathrm{ml}$ ethanol was slowly added to the sample, which was precipitated overnight. The FITC-Ficoll was centrifuged (1000 rpm for $15 \mathrm{~min}$ ) and the pellet resolved in $2 \mathrm{ml}$ Phosphate buffer. $\mathrm{pH}$ was adjusted to 6.5-7.0. The labeled Ficoll was purified from free FITC on a desalting column (Amersham Biosciences, Sephadex G-25 PD-10).

${ }^{125}$ I-labeled human serum albumin was obtained from Isopharma. The level of free tracer in the solution was less than $0.3 \%$, as determined after precipitation using $10 \%$ trichloroacetic acid. IgM was labeled by the iodogen method. $1 \mathrm{mg}$ of IgM was labeled with $300 \mu \mathrm{g}$ of iodogen. After labeling, the 
product was purified on a PD-10 column. A $2 \mathrm{ml}$ fraction was separated with a purity of $97.1 \%$. The specific activity was $29.5 \mathrm{MBq} / \mathrm{mg}$. Based on the HPLC pattern, there were no signs of fragmentation or formation of dimers. The level of free tracer in the ${ }^{131} \mathrm{I}-\mathrm{IgM}$ was less than $0.5 \%$.

To reduce the level of free tracer and possible fragments of labeled substances, all radiolabeled proteins were centrifuged prior to the intravascular administration on Amicon YM-30 filters (Millipore Corp., Bedford, MA) with a molecular cutoff of $30 \mathrm{kDa}$.

\section{Analysis of blood and dialysate}

Prior to measurements of radioactivity, all blood and dialysate samples were precipitated with $10 \%$ trichloroacetic acid to minimize the impact of free label. The samples were then centrifuged, the supernatant was removed (containing the free label), and the activity in the pellet was measured. Blood and dialysate samples were analyzed for radioactivity on a gamma counter (Wizard 1480; LKB-Wallac, Turku, Finland). Appropriate corrections for radioactive decay and spillover between the two isotope channels for ${ }^{125} \mathrm{I}$ and ${ }^{131} \mathrm{I}$ were done. Blood glucose concentrations were measured instantly on a Glucometer DEX 2 (Bayer AB, Göteborg, Sweden), while dialysate samples were analyzed for glucose concentration on an YSI 2300D (YSI Inc., Yellow Springs, OH).

\section{Analysis of Ficoll clearance}

43 minutes after the onset of the PD dwells, the mice were injected with a bolus dose (50 $\mu$ l) of a mixture (1:24) of FITC-Ficoll 400 (Sigma, St Louis, MO) and FITC-Ficoll 70 (Pharmacia, Uppsala, Sweden) of approximately $50 \mathrm{mg} / \mathrm{ml}$. A serum sample was drawn 27 minutes after the bolus injection of Ficoll. The plasma disappearance of Ficoll was more or less linear in the time interval for clearance measurements, and thus the midpoint plasma concentration could be used as an estimate of plasma Ficoll concentration. Dialysate was collected after approximately 46 minutes. Serum and dialysate samples were subjected to high-pressure size exclusion chromatography (HPSEC) using a system from Waters (Milford, MA) containing pump (Waters 1525), absorbance detector (Waters 2487), and a fluorescence detector (Waters 2475). The samples were analyzed at an excitation wavelength of $492 \mathrm{~nm}$ and an emission wavelength of $518 \mathrm{~nm}$. The system was controlled using the Breeze software (Waters). Size exclusion was achieved with 
an Ultrahydrogel-500 column (Waters), using a $0.05 \mathrm{M}$ Phosphate buffer with $0.15 \mathrm{M} \mathrm{NaCl}(\mathrm{pH} 7.4$ ) as mobile phase. The column was calibrated with narrow Ficoll standards ( $73 \AA, 59 \AA, 46 \AA, 38 \AA$ and $30 \AA)$ kindly provided by Dr Torvald Andersson (Pharmacia). Ficoll dialysate/plasma ratio (D/P) was calculated by dividing the dialysate concentration from the HPSEC with the average plasma concentration (plasma after 27 minutes of Ficoll exposure, corresponding to the mean plasma concentration over the experimental period). Ficoll clearance was then calculated by multiplying the $\mathrm{D} / \mathrm{P}$ with the average intraperitoneal volume and dividing by the experimental time.

\section{Electron microscopy and morphology}

After PD and under isofluran anesthesia, phosphate buffered saline (PBS) was rapidly infused through the carotid artery until fluid in the outflow catheter was blood-free in two controls and two cav-1-KO mice. Thereafter, $20 \mathrm{ml}$ fixation solution (PBS, $1 \%$ formaldehyde and $2.5 \%$ glutaraldehyde) was infused. The abdomen was cut open and the abdominal wall was carefully dissected and placed in vials with fixation solution. Preparations were kept at $4{ }^{\circ} \mathrm{C}$ over night and moved to the same solution without glutaraldehyde, in which they were kept until they were post-fixed for $1 \mathrm{~h}$ using osmium tetroxide. After dehydration in graded ethanols, the tissues were embedded in Durcupan and ultrathin sections were cut and contrasted with uranyl acetate and lead citrate. Digital images were obtained in a transmission electron microscope.

\section{Calculations and statistical analysis}

We have chosen to interpret our data in terms of the two-pore model (23), which is relatively simple and straightforward, though other more sophisticated models of capillary permselectivity exist (5). The twopore model is used daily in software designed to predict and describe the peritoneal exchanges in patients during PD worldwide. Details regarding the two-pore fit and the fiber-matrix-pore model are given in the appendix. Clearance calculations were performed as described in previous publications $(2,3,25,27)$ from the mass transfer of tracer per unit time divided by the average plasma concentration of tracer. PS for glucose was averaged from sequential measurements throughout the dwell, setting the sieving coefficient at 0.57 . All data are expressed as mean values \pm SEM. Statistical differences were analyzed using MannWhitney test on the computer program SPSS 11.0.1 for Macintosh OSX (SPSS Inc., Chicago, IL). 


\section{Results}

There were no differences between control and KO-mice regarding hemodynamic parameters. MAP was $76 \pm 3 \mathrm{mmHg}$ for control and $73 \pm 6 \mathrm{mmHg}$ for cav-1-KO mice, while HR was $550 \pm 14$ beats per minute (bpm) and $549 \pm 15 \mathrm{bpm}$, respectively.

There was an abundance of caveolae in the capillary endothelial cells from the abdominal wall of control mice (Fig. 1) as shown using electron microscopy. However, in the cav-1-KO mice caveolae were absent. Adherence junctions between capillary endothelial cells had a similar appearance in the sections examined from control and cav-1-KO mice.

The transperitoneal clearances of albumin and $\operatorname{IgM}$ in control and cav-1-KO mice are presented in Fig. 2 and Fig. 3. Protein transport was clearly dependent on molecular size. Cav-1-KO mice displayed a higher transvascular protein transport as compared to control mice. Thus, the albumin clearance was $0.088 \pm 0.008 \mu \mathrm{l} / \mathrm{min}$ and $0.179 \pm 0.012 \mu \mathrm{l} / \mathrm{min}(\mathrm{p}=0.001)$ in control and cav-1-KO mice, respectively (Fig. 2). The IgM clearance was $0.049 \pm 0.003 \mu \mathrm{l} / \mathrm{min}$ and $0.083 \pm 0.010 \mu \mathrm{l} / \mathrm{min}(\mathrm{p}=0.016)$ in cav-1-KO and control, respectively (Fig. 3). The transperitoneal Ficoll permeability pattern is presented on a semilogarithmic plot in Fig. 4. This pattern is clearly displaying a bimodal size selectivity. The Ficoll clearance patterns in control and cav-1-KO mice were more or less parallel, albeit with an upward shift for the cav-1-KO mice.

A two-pore analysis of the Ficoll data resulted in a small pore radius $\left(\mathrm{r}_{\mathrm{s}}\right)$ of $47 \pm 2 \AA$ for control mice, while $r_{s}$ was $45 \pm 1 \AA$ for the cav-1-KO mice (n.s.). The large pore radii $\left(r_{L}\right)$ were $183 \pm 11 \AA$ and $150 \pm 14 \AA$ in control and cav-1-KO, respectively (n.s.). The fractional hydraulic conductivity accounted for by the large pores $\left(\alpha_{\mathrm{L}}\right)$ was $0.021 \pm 0.002$ in control and $0.017 \pm 0.003$ in cav-1-KO (n.s.). However, the large pore fluid flow $\left(\mathrm{J}_{\mathrm{VL}}\right)$ was doubled $(\mathrm{p}=0.005)$ in cav-1-KO mice $(0.20 \pm 0.03 \mu 1 / \mathrm{min})$ as compared to control $(0.07 \pm 0.01 \mu 1 / \mathrm{min})$.

Small molecular transfer, as measured by PS for glucose, was $0.049 \pm 0.003 \mathrm{ml} / \mathrm{min}$ and $0.053 \pm 0.003$ $\mathrm{ml} / \mathrm{min}$ in control and cav-1-KO mice, respectively (n.s.). 


\section{Discussion}

The transperitoneal clearances of macromolecules (albumin and $\operatorname{IgM}$ ) were higher in mice lacking caveolae than in control mice, and there was a clear size-dependence of the transport (Figs. 2-3). The transperitoneal permeability pattern of Ficoll had typical bimodal size selectivity characteristics (Fig. 4). However, there was a parallel upward shift in the permeability for the cav-1-KO mice as compared to control mice. The small molecular transfer, i.e. PS for glucose, did not differ between control and cav-1KO mice. All in all, these data imply that macromolecules are transported through a passive porous pathway in both $\mathrm{KO}$ and control mice, but not by transcytosis.

The transport of proteins (albumin and IgM) and high molecular weight Ficoll was significantly higher in the cav-1-KO mice as compared to control in the present study (Figs. 2-4). Schubert et al. (39) also concluded that a higher albumin transport is present in cav-1-KO mice, using different methodology. The higher macromolecular clearances in cav-1-KO mice can be interpreted in four ways; 1) A newly formed, alternative paracellular pathway is opened in cav-1-KO mice, 2) the number of large pores in a preexisting two-pore system is increased, 3) the large pore volume flow in this system is increased (23), or 4) the selectivity of the endothelial glycocalyx to solute transport is reduced (5).

Previous reports $(6,39)$, regarding the transvascular transport rates of macromolecules, have speculated that the high transport rate in cav-1-KO mice was due to the appearance of an "alternative" paracellular route. IgM transport through "normal” large pores $(\approx 200 \AA$ radii) is relatively restricted. On the contrary, albumin transport through these pores is only restricted to a minor degree. Hence, the opening of a new large diameter route would affect the largest molecule (IgM) to a greater extent than albumin (23). This was not observed. In the same manner, the relative difference in clearance for small and large Ficolls would be smaller, giving rise to a more flat permeability profile in the high molecular weight portion. Again, this pattern was not seen for the Ficoll clearance data. Furthermore, the opening of a new paracellular pathway would impact on the large pore radii. The two-pore analysis did not reveal different large pore radii for cav-1-KO mice as compared to control, however. Thus, it is unlikely that a new paracellular pathway had appeared in cav-1-KO mice. 
Another parameter obtained by the two-pore analysis is the fractional hydraulic conductivity through large pores $\left(\alpha_{\mathrm{L}}\right)$. Increasing the frequency of large pores would increase the $\alpha_{\mathrm{L}}$, and lead to increased filtration of macromolecules. However, the $\alpha_{\mathrm{L}}$ in control and cav-1-KO mice were not significantly different according to the two-pore analysis. Thus, it is unlikely that the increased protein transport in cav1-KO mice is due to an increased number of large pores.

The third possibility is that the large pore volume flow $\left(\mathrm{J}_{\mathrm{vL}}\right)$ may be increased, giving rise to the higher macromolecular transport. Indeed, the two-pore analysis revealed a significantly higher $\mathrm{J}_{\mathrm{vL}}$ $(\mathrm{p}=0.005)$ in cav-1-KO as compared to control $(0.20 \pm 0.03 \mu \mathrm{l} / \mathrm{min}$ vs. $0.08 \pm 0.009 \mu \mathrm{l} / \mathrm{min})$. Although the mechanism is unknown, a possible explanation could be the higher level of nitric oxide (NO) due to alleviation of an inhibitory influence of caveolin-1 on endothelial NO-synthase (eNOS) (6). NO is a wellknown vasodilator $(9,16)$, and the higher level of NO may give rise to vasodilatation in resistance vessels and thus a higher capillary hydraulic pressure, and consequently, an increased $\mathrm{J}_{\mathrm{vL}}$. Thus, more macromolecules would be transported by convection across the large pores.

We have chosen a pore model to describe peritoneal permeability in control and cav-1-KO mice, since this is the "classical" and most simple and straightforward way of describing membrane transport data. Furthermore, a heteroporous membrane model (the so-called three-pore model) has been extensively and successfully used in conjunction with PD to model solute and fluid transport as a function of variations in dialysate osmotic agent (26), dialysate ion composition (31), and variations in a number of physiological parameters affecting transport (29). An alternative way of describing the data is by applying a fiber-matrix-pore model (5). In this model the main size-selective structures are within the glycocalyx, covering the endothelial cells on the luminal side. The rationale for this approach is that NO may affect essential structures within the endothelial glycocalyx (7), and thus represents the fourth possibility of explaining the observed transport pattern. We have modeled a fiber-matrix coupled in series with interendothelial slits or pores as a "pre-filter". In the simplest variant of this model, the slits or pores are almost completely non-restrictive, and the main size-selectivity is within the matrix itself (11). A more detailed description of the analysis and the outcome is given in the appendix. Applying this model to the present data yields an almost perfect fit of the model to experimental Ficoll clearance data vs. molecular 
radius $\left(a_{\mathrm{e}}\right)$. A relatively small reduction in fiber density was found to result in a near doubling of the hydraulic conductance of the matrix and a moderately reduced selectivity. Across the low-density fiber region these alterations can fully explain the increased apparent fluid flow, and macromolecular filtration, through the less dense region. Thus, if capillary size-selectivity is assumed to reside mainly in the glycocalyx, a rather moderate change in glycocalyx density can explain the cav-1-KO data without assuming any accompanying changes in microvascular pressure.

In the electron-microscopic sections, there was an abundance of caveolae in wild-type mice in abdominal wall capillary endothelial cells (Fig. 1), whereas in the cav-1-KO mice there was a complete lack of endothelial vesicles. Furthermore, the tight junction morphology displayed no signs of abnormalities in any of the sections examined. Thus, quantitative clearance data, modeling of measured data, and morphologic data fail to support the appearance of an alternative pathway or any abnormality in tight junctions in cav-1-KO mice.

The capillaries involved in peritoneal exchange have continuous capillary walls. This type of capillary bed is prominent throughout the body and responsible for exchanges in tissues such as musculature, connective tissue, and adipose tissue. A large portion of the total cardiac output is circulated through continuous capillary beds. The values of tracer loss from plasma reported by Schubert and coworkers (39) reflect the whole animal, and all types of vascular beds, including leaky fenestrated capillary beds such as in secretory glands, liver, viscera, and kidney (that is more or less impermeable to molecules such as albumin). Given the higher basal level of NO in cav-1-KO mice, a lower blood pressure may be expected in these mice. However, the systemic mean arterial pressure (MAP) was almost identical in control and cav-1-KO mice. Furthermore, the heart rate did not differ between control and cav-1-KO mice. Cav-1-KO mice may, nonetheless, have a higher capillary pressure in the absence of a higher MAP due to NOmediated precapillary vasodilatation.

The two-pore model does not take into account the distribution of vessels within the tissue, and large molecules are somewhat restricted in their passage through the interstitium. Across a double-layered capillary-interstitial serial barrier, the total transport resistance is largely determined by the barrier that offers the highest resistance (25). For macromolecules, the resistance across the capillary wall is 
tremendous in comparison to the resistance across the interstitium (25). Furthermore, no obvious signs of a modified interstitium could be seen in the EM sections taken from both strains. Moreover, the transperitoneal transport of small molecules, such as glucose, may actually be modified by the interstitium (34). For small solutes, the interstitium may thus add a significant transport resistance, and a change in the interstitial dimensions may impact on the transport of these. This was not seen. It is therefore not likely that a change in the interstitium has contributed to the increased large solute transport in cav-1-KO mice.

In a previous paper, Schubert et al. (39) attempted to investigate microvascular transport of albumin in cav-1-KO mice. As in the present study, the authors found a higher transport of albumin in KO mice and postulated that this was due to the de novo appearance of a "compensatory pathway" in cav-1-KO mice. Their method was simply to inject ${ }^{125}$ I-labeled albumin in the tail vein of the mice and then to collect blood samples at a few time points up to $60 \mathrm{~min}$. The method represents a measurement of tracer disappearance from the plasma compartment and not a quantification of clearance. The injected volume in a mouse must not exceed $50 \mu 1(5 \%$ of plasma volume $)$ since the total plasma volume is very small $(\approx 1$ ml). The injection artifact induced by Schubert and coworkers was, however, substantial due to the large volume injected $(150 \mu \mathrm{l})$ and was different among control and cav-1-KO mice. Furthermore, Schubert et al. measured the uptake of radiolabeled albumin to various tissues [Fig. 2, (39)]. In tissue uptake studies of macromolecules it is pivotal to remove all remaining tracer from the vascular compartment (by a wash-out procedure) since most of the macromolecule distribution is within the vasculature. This can only be achieved by vascular perfusion and washout. Schubert and coworkers, however, only washed the dissected tissues "thoroughly" in phosphate-buffered saline. Under these circumstances, the measured tissue uptake will reflect the amount of tracer remaining in the vascular compartment. Hence, all of the most blood-rich tissues showed the highest accumulation in the paper by Schubert et al. Since the cav-1 KO mice may be more vasodilated due to the increased NO-levels, their tissues are expected to have a higher blood content.

Schubert and coworkers speculated that the "hyperpermeability" of the cav-1-KO mice may be due to the presence of high NO levels. However, the effect of NO on microvascular permeability is subject to great controversy (10). In vitro studies with blood-free perfusion have shown an increased capillary permeability induced by NO (10), while in vivo studies using blood perfusion consistently demonstrate a 
reduced microvascular permeability by NO in physiological concentrations $(10,17,18)$. To support their contention of increased permeability due to $\mathrm{NO}$, Schubert et al. repeated their experiments on albumin disappearance with or without pretreating the mice with L-nitro-arginine methyl ester (L-NAME), which is a known inhibitor of eNOS. The albumin disappearance from plasma was reduced in KO animals after L-NAME, but no effect was seen for the wild type mice. However, these results can also be explained by precapillary vasodilatation by NO, as discussed above. Thus, cav-1-KO mice may increase their vascular tone due to the inhibitory effect of L-NAME, and as a consequence, the capillary pressure will decrease and thus the $\mathrm{J}_{\mathrm{vL}}$, giving rise to a reduced albumin clearance. That the acute application of L-NAME would lead to a rapid (within minutes) growth of the endothelial glycocalyx, one of the tentative explanations of the altered clearance in the cav-1-KO mice, seems less likely, but cannot be entirely ruled out.

To summarize, we found no evidence that transcytosis, mediated by caveolae, would serve as a quantitatively important mode of the transport of albumin, IgM or Ficoll across the endothelium in vivo. All in all, our data contradict the contention of active transport of bulk proteins across the peritoneal capillaries in vivo, as well as the concept of an alternative transport pathway in cav-1-KO mice. Rather they are consistent with passive, convective protein transport through large pores in the microvascular walls. Macromolecular transport was increased in cav-1-KO mice, conceivably due to precapillary vasodilatation, caused by an increased eNOS activity in mice lacking cav-1, giving rise to an increased capillary hydrostatic pressure, and thus, a higher filtration rate of macromolecules through large pores. Alternatively, the endothelial glycocalyx may have been modified due to the high NO levels in cav-1-KO mice, giving rise to an increased capillary hydraulic conductivity and an enhanced transvascular filtration of macromolecules. 


\section{Acknowledgements}

This study was supported by grants from the Swedish Medical Research Council (grant no 14X-08285 and 71X-14955), from the Åke Wiberg foundation, from the Knut and Alice Wallenberg foundation, from the Lars Hierta foundation, and by a grant from the Ragnar Söderberg Foundation to the Vascular Wall Program at Lund University. The secretarial assistance by Kerstin Wihlborg is gratefully acknowledged. Jonas Broman is acknowledged for assistance with EM and Tomas Ohlsson for labeling and analysis of tracers. 


\section{Appendix}

Two-pore fit

The unidirectional clearance data $(\mathrm{Cl})$ were fitted to a two-pore model of membrane permeability (23) using a non-linear least squares regression analysis. In detail, the function to be minimized was:

$$
\mathrm{SSQ}=\sum_{\mathrm{a}_{\mathrm{e}}=20}^{100}\left(\frac{\mathrm{Cl}_{\text {exp }}\left(\mathrm{a}_{\mathrm{e}}\right)-\mathrm{Cl}_{\mathrm{th}}\left(\mathrm{a}_{\mathrm{e}}\right)}{\mathrm{Cl}_{\text {exp }}\left(\mathrm{a}_{\mathrm{e}}\right)}\right)^{2}
$$

where the subscripts exp and th refer to the experimentally and theoretically calculated $\mathrm{Cl}$, respectively, and the sum is extended to the Stokes-Einstein radii $\left(\mathrm{a}_{\mathrm{e}}\right)$ from 20 to $100 \AA$. The relative difference with respect to $\mathrm{Cl}_{\text {exp }}$ was introduced in place of the usual squared difference, to compensate for the large range of experimental values (two orders of magnitude in $\mathrm{Cl}_{\exp }$ going from the minimum to the maximum $\mathrm{a}_{\mathrm{e}}$ ).

The estimated parameters were: the small and large pore radii $\left(r_{s}\right.$ and $r_{L}$, respectively), the fractional hydraulic conductance accounted for by the large pores $\left(\alpha_{\mathrm{L}}\right)$, and the fluid flow through the large pores $\left(\mathrm{J}_{\mathrm{VL}}\right)$. However, because the numerical values of these parameters differ by several orders of magnitude (six from $r_{L}$ to $\alpha_{L}$ ), a set of scaling multipliers was introduced, so that the minimization algorithm had to deal with parameters near to unity.

\section{Fiber-matrix-pore model fit}

For comparison with the two-pore modeling, we have also made a fit of average Ficoll clearance data vs. molecular radii $\left(\mathrm{a}_{\mathrm{e}}\right)$ using a bimodal selectivity fiber matrix model, as described in detail by Michel (11). The fiber radius was set at $5 \AA$, and the fiber density was assumed to be essentially homogeneous, except for approximately $1 \%$ of the matrix, showing a lower density. Fiber densities (one minus the void volume) of the "dense" and "loose" regions were adapted to match experimental data in control and, after allowing for a slightly increased volume flow through the loose regions, to the corresponding data in cav-1-KO mice. Thus, to model the data for control mice, a fractional fiber volume of $5.5 \%$ (void volume 0.945 ) and a fiber radius of $5 \AA$ were applied. A small fraction of the surface area ( $\sim 1 \%$ of the fiber-matrix) accounting for $\sim 0.06 \mu \mathrm{l} / \mathrm{min}$ of the total fluid flow, with a density of $0.4 \%$, had also to be added to fit the data at high molecular radii $\left(a_{e}>50 \AA\right)$. In cav-1-KO mice the data were best described if the fractional 
fiber volume was reduced to $4.8 \%$ (void volume 0.952 ) for a fluid flow through the low-density matrix regions of $\sim 0.07 \mu 1 / \mathrm{min}$, where the fiber density was reduced to $0.3 \%$. 


\section{References}

1. Bundgaard M. The three-dimensional organization of tight junctions in a capillary endothelium revealed by serial-section electron microscopy. J Ultrastruct Res 88: 1-17, 1984.

2. Carlsson O, Nielsen S, Zakaria el R, and Rippe B. In vivo inhibition of transcellular water channels (aquaporin-1) during acute peritoneal dialysis in rats. Am J Physiol 271: H2254-H2262, 1996.

3. Carlsson O, Rosengren BI, and Rippe B. Effects of peritoneal hyaluronidase treatment on transperitoneal solute and fluid transport in the rat. Acta Physiol Scand 168: 371-376, 2000.

4. Carlsson O, Rosengren BI, and Rippe B. Transcytosis inhibitor N-ethylmaleimide increases microvascular permeability in rat muscle. Am J Physiol Heart Circ Physiol 281: H1728-H1733, 2001.

5. Curry FR. Microvascular solute and water transport. Microcirculation 12: 17-31, 2005.

6. Drab M, Verkade P, Elger M, Kasper M, Lohn M, Lauterbach B, Menne J, Lindschau C, Mende F, Luft FC, Schedl A, Haller H, and Kurzchalia TV. Loss of caveolae, vascular dysfunction, and pulmonary defects in caveolin-1 gene-disrupted mice. Science 293: 2449-2452, 2001.

7. Fransson LA, Belting M, Cheng F, Jonsson M, Mani K, and Sandgren S. Novel aspects of glypican glycobiology. Cell Mol Life Sci 61: 1016-1024, 2004.

8. Frokjaer-Jensen J. Three-dimensional organization of plasmalemmal vesicles in endothelial cells. An analysis by serial sectioning of frog mesenteric capillaries. J Ultrastruct Res 73: 9-20, 1980.

9. Ignarro LJ, Buga GM, Wood KS, Byrns RE, and Chaudhuri G. Endothelium-derived relaxing factor produced and released from artery and vein is nitric oxide. Proc Natl Acad Sci U S A 84: 92659269, 1987.

10. Kubes P. Nitric oxide affects microvascular permeability in the intact and inflamed vasculature. Microcirculation 2: 235-244, 1995.

11. Michel CC. Filtration coefficients and osmotic reflexion coefficients of the walls of single frog mesenteric capillaries. J Physiol 309: 341-355, 1980.

12. Michel CC and Curry FE. Microvascular permeability. Physiol Rev 79: 703-761, 1999. 
13. Ogawa K, Imai M, Ogawa T, Tsukamoto Y, and Sasaki F. Caveolar and intercellular channels provide major transport pathways of macromolecules across vascular endothelial cells. Anat Rec 264: $32-$ $42,2001$.

14. Palade GE. Fine Structure of Blood Capillaries. J Appl Phys 24: 1424-1424, 1953.

15. Palade GE. Transport in quanta across the endothelium of blood capillaries. Anat Rec 136: 254-254, 1960.

16. Palmer RM, Ferrige AG, and Moncada S. Nitric oxide release accounts for the biological activity of endothelium-derived relaxing factor. Nature 327: 524-526, 1987.

17. Persson J, Ekelund U, and Grände PO. Endogenous nitric oxide reduces microvascular permeability and tissue oedema during exercise in cat skeletal muscle. $J$ Vasc Res 40: 538-546, 2003.

18. Persson J, Ekelund U, and Grände PO. Nitric oxide and prostacyclin play a role in the regulation of microvascular protein and hydraulic permeability in cat skeletal muscle. Microcirculation 10: 233-243, 2003.

19. Predescu D, Horvat R, Predescu S, and Palade GE. Transcytosis in the continuous endothelium of the myocardial microvasculature is inhibited by N-ethylmaleimide. Proc Natl Acad Sci U S A 91: 30143018, 1994.

20. Predescu D and Palade GE. Plasmalemmal vesicles represent the large pore system of continuous microvascular endothelium. Am J Physiol 265: H725-H733, 1993.

21. Predescu D, Predescu S, McQuistan T, and Palade GE. Transcytosis of alpha1-acidic glycoprotein in the continuous microvascular endothelium. Proc Natl Acad Sci U S A 95: 6175-6180, 1998.

22. Predescu SA, Predescu DN, and Palade GE. Plasmalemmal vesicles function as transcytotic carriers for small proteins in the continuous endothelium. Am J Physiol 272: H937-H949, 1997.

23. Rippe B and Haraldsson B. Transport of macromolecules across microvascular walls: the two-pore theory. Physiol Rev 74: 163-219, 1994.

24. Rippe B, Kamiya A, and Folkow B. Transcapillary passage of albumin, effects of tissue cooling and of increases in filtration and plasma colloid osmotic pressure. Acta Physiol Scand 105: 171-187, 1979. 
25. Rippe B and Krediet RT. Peritoneal physiology-Transport of solutes. In: The Textbook of Peritoneal Dialysis (1 ed.), edited by Gokal R and Nolph KD. Dordrecht: Kluwer Academic Publishers, 1994, p. 69113.

26. Rippe B and Levin L. Computer simulations of ultrafiltration profiles for an icodextrin-based peritoneal fluid in CAPD. Kidney Int 57: 2546-2556, 2000.

27. Rippe B, Rosengren BI, Carlsson O, and Venturoli D. Transendothelial transport: the vesicle controversy. J Vasc Res 39: 375-390, 2002.

28. Rippe B, Rosengren BI, and Venturoli D. The peritoneal microcirculation in peritoneal dialysis. Microcirculation 8: 303-320, 2001.

29. Rippe B, Stelin G, and Haraldsson B. Computer simulations of peritoneal fluid transport in CAPD. Kidney Int 40: 315-325, 1991.

30. Rippe B and Taylor A. NEM and filipin increase albumin transport in lung microvessels. Am $J$ Physiol Heart Circ Physiol 280: H34-H41, 2001.

31. Rippe B, Venturoli D, Simonsen O, and de Arteaga J. Fluid and electrolyte transport across the peritoneal membrane during CAPD according to the three-pore model. Perit Dial Int 24: 10-27, 2004.

32. Rosengren BI, Al Rayyes O, and Rippe B. Transendothelial transport of low-density lipoprotein and albumin across the rat peritoneum in vivo: effects of the transcytosis inhibitors NEM and filipin. $J$ Vasc Res 39: 230-237, 2002.

33. Rosengren BI, Carlsson O, Venturoli D, Al Rayyes O, and Rippe B. Transvascular Passage of Macromolecules into the Peritoneal Cavity of Normo- and Hypothermic Rats in vivo: Active or Passive Transport? J Vasc Res 41: 123-130, 2004.

34. Rosengren BI and Rippe B. Blood flow limitation in vivo of small solute transfer during peritoneal dialysis in rats. J Am Soc Nephrol 14: 1599-1604, 2003.

35. Rutledge JC. Temperature and hydrostatic pressure-dependent pathways of low-density lipoprotein transport across microvascular barrier. Am J Physiol 262: H234-H245, 1992.

36. Rutledge JC, Curry FE, Blanche P, and Krauss RM. Solvent drag of LDL across mammalian endothelial barriers with increased permeability. Am J Physiol 268: H1982-H1991, 1995. 
37. Schnitzer JE, Allard J, and Oh P. NEM inhibits transcytosis, endocytosis, and capillary permeability: implication of caveolae fusion in endothelia. Am J Physiol 268: H48-H55, 1995.

38. Schnitzer JE, Oh P, Pinney E, and Allard J. Filipin-sensitive caveolae-mediated transport in endothelium: reduced transcytosis, scavenger endocytosis, and capillary permeability of select macromolecules. J Cell Biol 127: 1217-1232, 1994.

39. Schubert W, Frank PG, Woodman SE, Hyogo H, Cohen DE, Chow CW, and Lisanti MP. Microvascular hyperpermeability in caveolin-1 (-/-) knock-out mice. Treatment with a specific nitricoxide synthase inhibitor, L-name, restores normal microvascular permeability in Cav-1 null mice. $J$ Biol Chem 277: 40091-40098, 2002.

40. Zakaria ER and Rippe B. Intraperitoneal fluid volume changes during peritoneal dialysis in the rat: indicator dilution vs. volumetric measurements. Blood Purif 13: 255-270, 1995. 


\section{Figure legends}

Figure 1. Electron micrographs of capillary endothelial cells from the abdominal wall. Areas in boxes in upper figures are enlarged $3 \mathrm{X}$ in bottom figures. Numerous caveolae (indicated by arrows) were found in the control animals. Endothelial cells in KO-mice were completely devoid of caveolae. Scale bars $500 \mathrm{~nm}$.

Figure 2. Transperitoneal clearance $( \pm \operatorname{SEM})$ of albumin in control $(n=8)$ and cav-1-KO mice $(n=8)$. There was a significantly higher albumin clearance in cav-1-KO mice as compared to control $(\mathrm{p}=0.001)$.

Figure 3. Transperitoneal clearance $( \pm S E M)$ of $\operatorname{IgM}$ in control $(n=8)$ and cav-1-KO mice $(n=8)$. There was a significantly higher IgM clearance in cav-1-KO mice as compared to control $(\mathrm{p}=0.016)$.

Figure 4. Semilogarithmic plot of unidirectional transperitoneal clearances of polydisperse FITC-Ficoll versus molecular radii for control mice (lower graph) and cav-1-KO mice (upper graph). The data had clear-cut bimodal size selectivity characteristics. There was a parallel upward shift of the data from cav-1KO mice. A two-pore analysis of the data resulted in small pore radii of $47 \pm 2 \AA$ and $45 \pm 1 \AA$ for control and cav-1-KO, respectively (not significantly different). The large pore radii fitting the data were $183 \pm 11$ $\AA$ and $150 \pm 14 \AA$ for control and cav-1-KO, respectively (not significantly different). The fractional hydraulic conductivity in large pores $\left(\alpha_{\mathrm{L}}\right)$ was $0.021 \pm 0.002$ in control, and $0.017 \pm 0.003$ in cav-1-KO

(n.s.). However, the large pore fluid flow $\left(\mathrm{J}_{\mathrm{VL}}\right)$ was more than twice $(\mathrm{p}=0.005)$ in cav-1-KO mice $(0.20 \pm 0.03 \mu \mathrm{l} / \mathrm{min})$ as compared to control $(0.07 \pm 0.01 \mu \mathrm{l} / \mathrm{min})$. Data $( \pm \mathrm{SEM})$ from control $(\mathrm{n}=8)$ and cav$1-\mathrm{KO}(\mathrm{n}=8)$. 
Figures

Figure 1

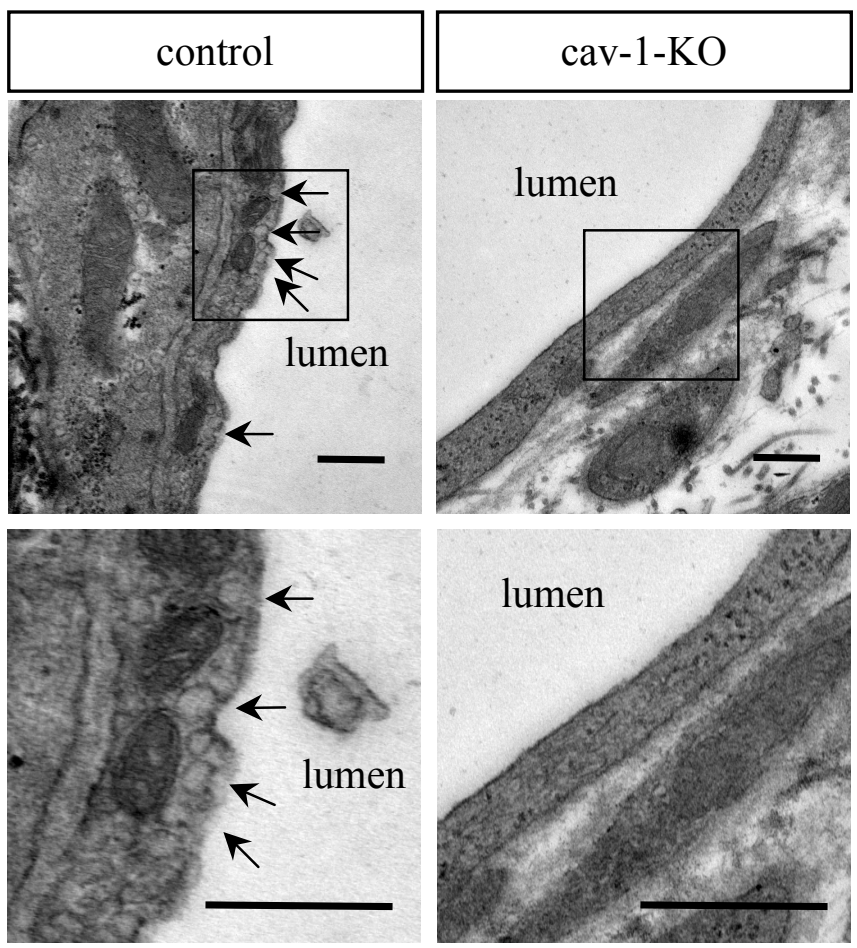


Figure 2

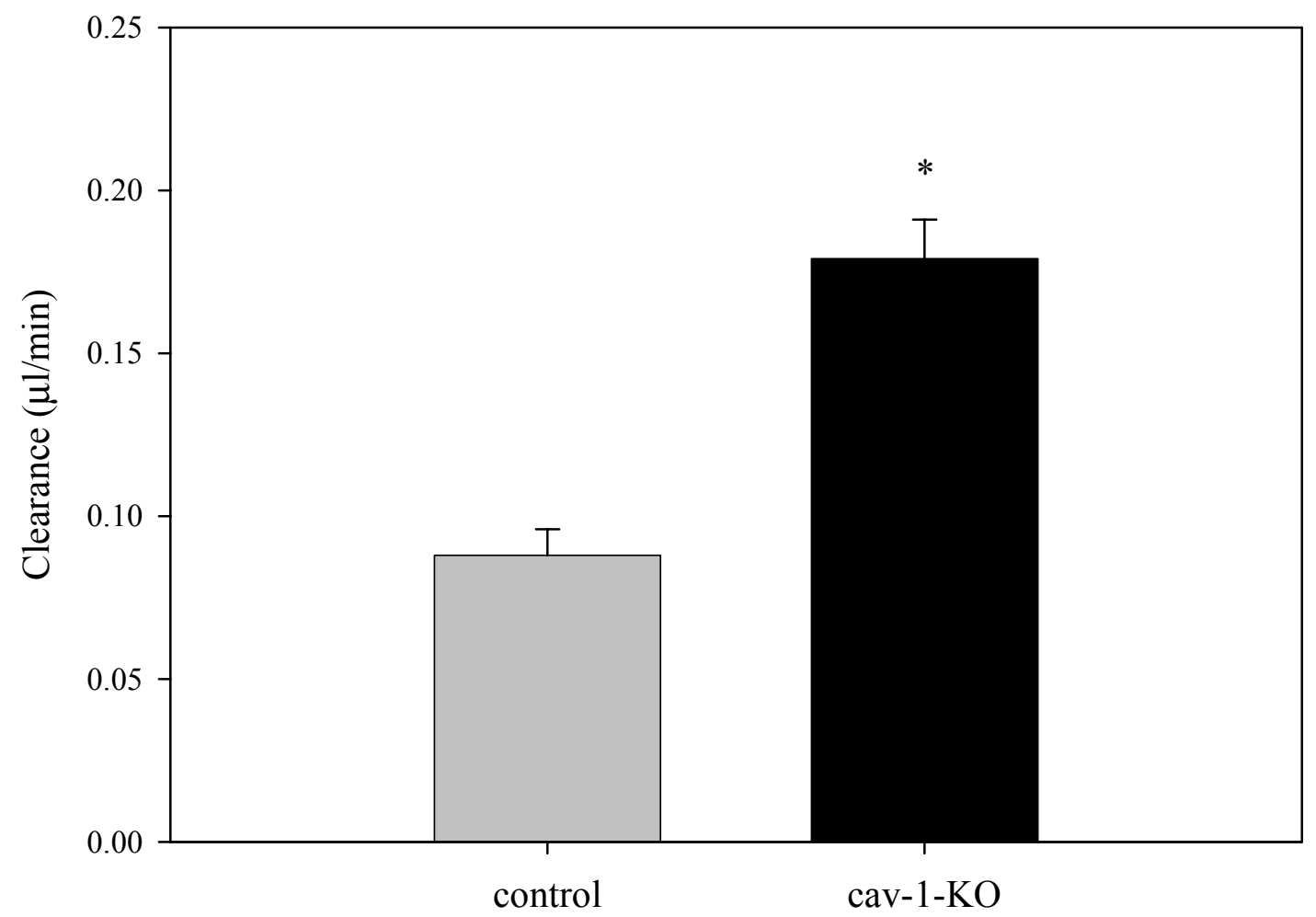


Figure 3.

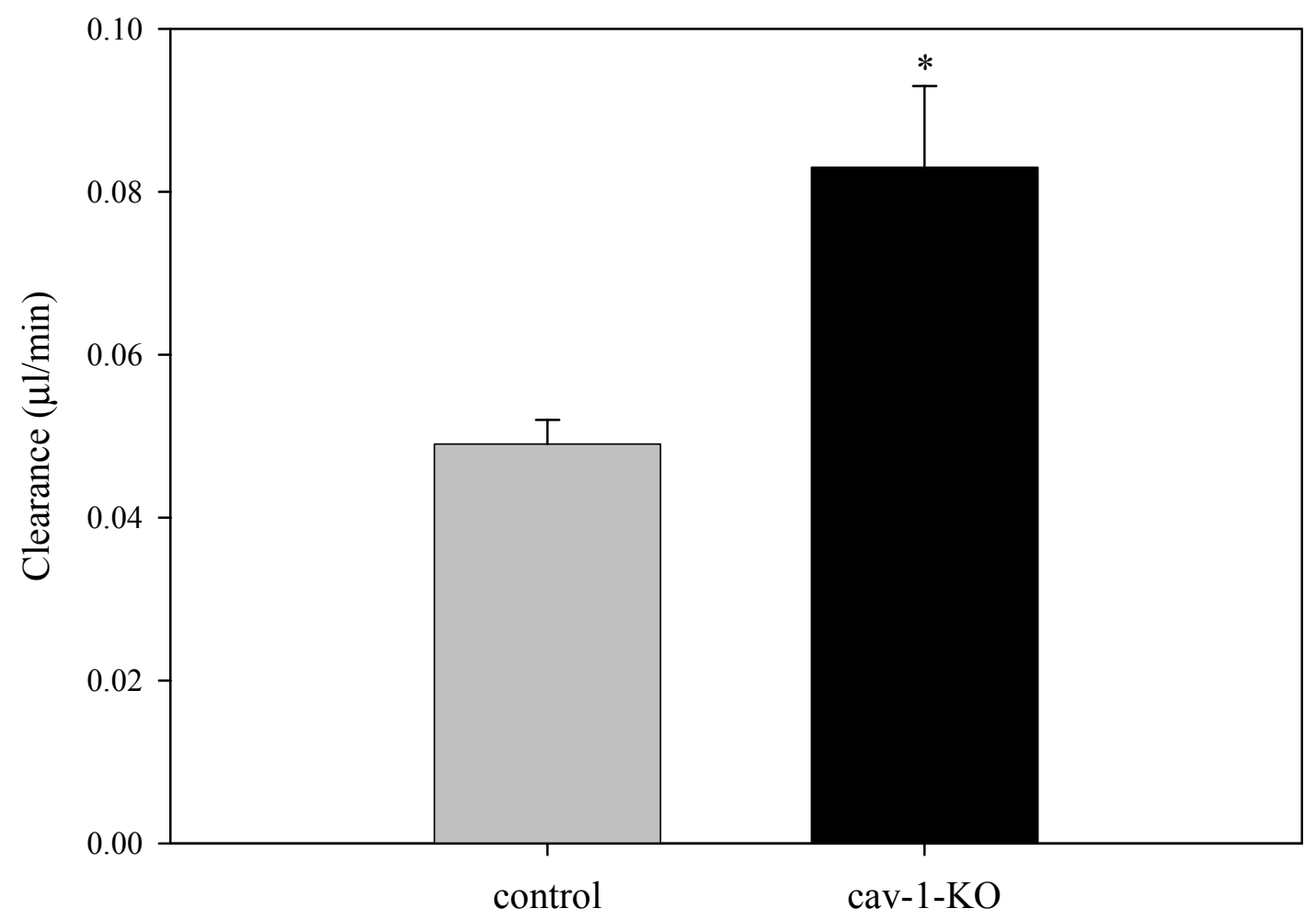


Figure 4.

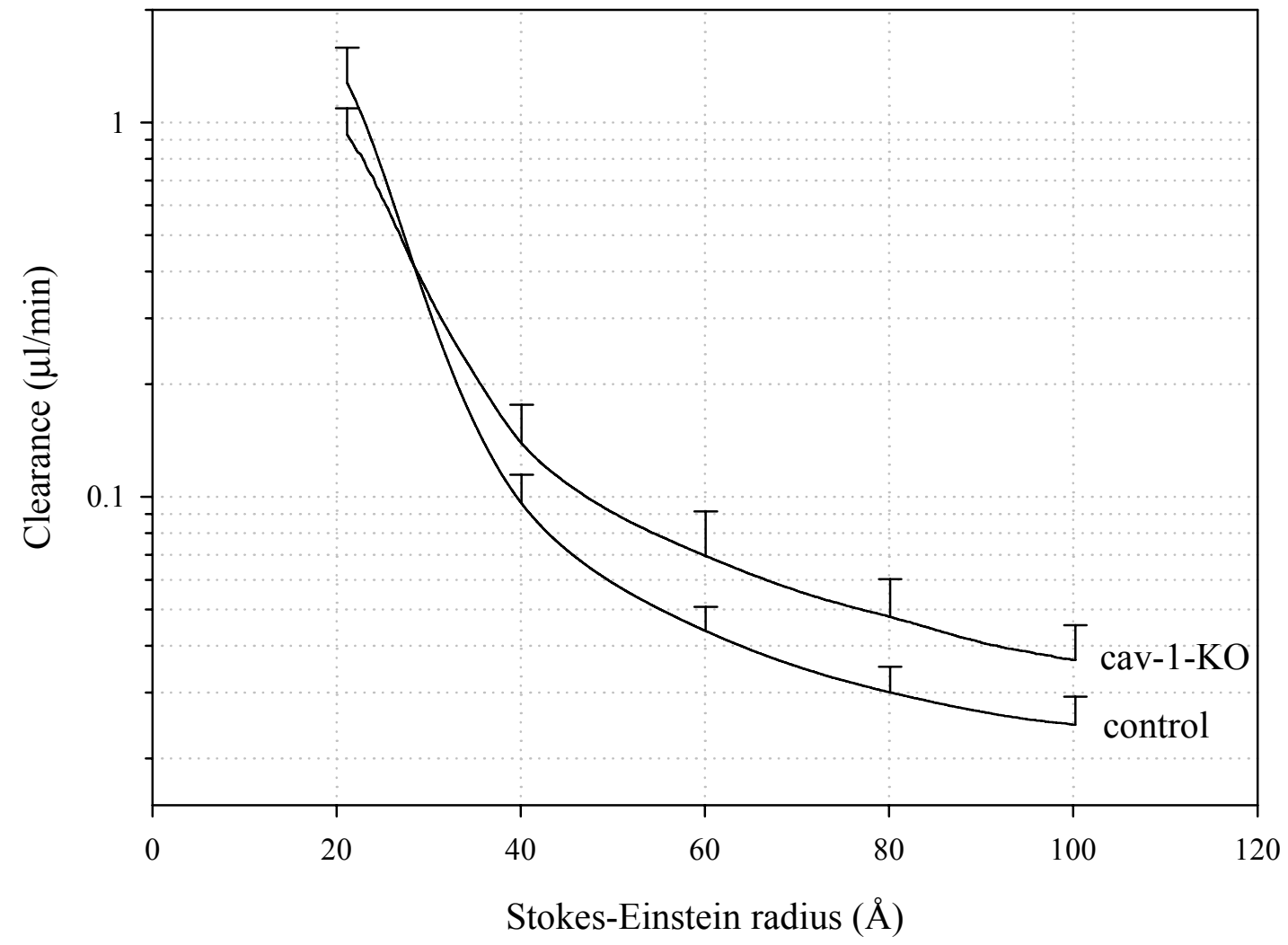

